\title{
¿Qué nos aporta la cirugía híbrida de revascularización miocárdica?
}

\author{
Carlos A. Mestres \\ FETCS \\ Cardiothoracic and Vascular Surgery. Heart and Vascular Institute \\ Cleveland Clinic Abu Dhabi \\ Abu Dhabi (Emiratos Arabes Unidos)
}

Introducción: El conocimiento actual nos indica que la cirugía de revascularización miocárdica (CRM) es el patrón-oro en el tratamiento de diversos grupos de pacientes afectos de cardiopatía isquémica (CI). Esto está bien definido en las recientes guías de práctica clínica conjuntas de la European Society of Cardiology (ESC) y la European Association for Cardio-thoracic Surgery $(\text { EACTS })^{1}$. El estudio "Synergy between Percutaneous Coronary Intervention with TAXUS and Cardiac Surgery (SYNTAX)" ha confirmado esta aserción y, a su vez, ha representado una revolución en el mundo de la Cardiología por la evidencia que ha producido con respecto al impacto positivo de la CRM, en especial en pacientes con anatomía compleja ${ }^{2}$. Las conclusiones son que la CRM debe seguir siendo el tratamiento estándar de los pacientes con lesiones complejas (puntuaciones
SYNTAX intermedias o altas). Para los pacientes con enfermedad menos compleja (puntuaciones SYNTAX bajas) o con enfermedad del tronco común (puntuaciones SYNTAX intermedias o bajas), la intervención coronaria percutánea (ICP) es una alternativa aceptable. Todos los pacientes con enfermedad coronaria multivaso deben ser revisados y discutidos por cirujanos y cardiólogos intervencionistas para alcanzar consenso con respecto al tratamiento óptimo ${ }^{2}$. Dicho esto, hay que añadir que los datos de SYNTAX confirman que el análisis de la causa de muerte confirmó que el fallecimiento cardíaco por infarto de miocardio es 10 veces más frecuente con la ICP que con la CRM en los pacientes de riesgo elevado. Esto ha sido discutido en una elegante y reciente contribución de Mack et $\mathrm{al}^{3}$, quienes indican que, además de lo antedicho, se confirmó una ventaja clara de la CRM en la

Correspondencia:

Carlos A. Mestres MD PhD FETCS

Cardiothoracic and Vascular Surgery

Heart and Vascular Institute

Cleveland Clinic Abu Dhabi

Al Maryah Island

112412 Abu Dhabi

(Emiratos Arabes Unidos)

MestreC@ClevelandClinicAbuDhabi.ae 
prevención de la muerte en los pacientes con enfermedad de tres vasos y puntuaciones SYNTAX intermedias y altas y en los pacientes con lesión de tronco común con puntuación SYNTAX alta. Asimismo, esta ventaja incremental era estadísticamente significativa y se ampliaba con el tiempo, lo cual debería ser comunicado de forma clara y transparente a los pacientes ${ }^{3}$; esto último es, por lo menos, infrecuente.

\section{Los hechos diferenciales}

La CRM tiene diversos hechos diferenciales que han sido confirmadas a lo largo de los años, con extensa documentación en la literatura, a saber:

1. La utilización de la arteria mamaria interna (AMI) (arteria torácica interna - ATI) en la revascularización del territorio de la arteria coronaria descendente anterior (DA).

2. La utilización de parada (plejía) cardíaca con soluciones hiperpotasémicas e hiperosomolares con diversos substratos metabólicos para conseguir un campo exangüe y facilitar la sutura quirúrgica de precisión.

Estos dos hechos han sido el motor de la CRM en las últimas cinco décadas. Las contribuciones palmarias de Cleveland Clinic contribuyeron a la definición del conocimiento actual sobre la CRM y sus resultados alejados. En concreto, la revascularización de la DA con la AMI izquierda es, probablemente, el hecho más importante a destacar ${ }^{4-5}$. Se podría argumentar que la variante de CRM sin circulación extracorpórea (CEC), es decir, con el corazón latiendo y sin el soporte mecánico de la maquina corazón-pulmón, aporta unos resultados similares, reduciendo la morbi-mortalidad intrínseca de la CEC. No obstante, y a pesar de las aportaciones y discusiones en la literatura quirúrgica, esto no se ha confirmado de modo y manera que la CRM sin CEC pueda recomendarse con clase IA a pesar de que un meta-análisis reciente sugiere que esta última estrategia pueda ser beneficiosa en pacientes de riesgo elevado ${ }^{6}$. Su papel parece estar bien definido $^{7}$.

\section{La Historia y la Evidencia}

Se cumplen ya 50 años de cirugía coronaria ${ }^{8-9}$ y la CRM con o sin CEC es una terapéutica establecida e inamovible en el tratamiento de le enfermedad coronaria de tronco común y multivaso. El conocimiento se ha definido a través de multitud de estudios de todo tipo, ob- servaciones, prospectivos, retrospectivos y aleatorizados A este respecto, los estudios $\mathrm{SYNTAX}^{2}$, ya comentado, ASCERT $^{10}$ y FREEDOM ${ }^{11}$ han comunicado resultados de supervivencia y eventos superiores de la CRM en comparación con la intervención coronaria percutánea (ICP). No hay que olvidar, sin embargo, el impacto asimismo positivo que ha tenido la ICP en subgrupos, también específicos, de pacientes como ocurre en la isquemia miocárdica aguda ${ }^{12}$ lo cual se ha traducido en cambios muy apreciables e importantes en los patrones de revascularización.

La historia también nos demuestra que modificaciones del procedimiento quirúrgico, como la introducción de un segundo injerto arterial en un territorio diferente a la DA y en grupos de pacientes seleccionados, aporta una mejoría adicional de los datos de supervivencia y control de eventos cardiacos alejados. Los datos de Lytle y cols ${ }^{13}$ también de Cleveland Clinic ya fueron ilustrativos en su momento. Y finalmente, la revascularización con múltiples injertos arteriales en sus diversas variantes como injertos secuenciales, con doble AMI o complementado con arteria radial, ofrecen ventajas de permeabilidad alejadas $^{14-15}$. Datos de reciente aparición confirman de nuevo el beneficio de la CRM con injerto arterial múltiple con respecto a la CRM convencional basada en el injerto de AMI izquierda y los dispositivos endocoronarios ${ }^{16}$. Esta es una práctica que debería incrementarse, aun conociendo que la selección de pacientes para todas las terapias y estrategias posibles debe ajustarse al paciente ${ }^{17}$. Un tema adicional en el ámbito quirúrgico es el de la CRM sin CEC. Discutirlo en profundidad puede llenar páginas de revistas científicas y libros de texto, pero la información reciente apoya con fuerza y claridad el papel fundamental de la estrategia de CRM con CEC para conseguir una revascularización completa. Y este es el segundo caballo de batalla en la CRM, es decir, revascularizar todos los vasos posibles que tengan lesiones consideradas como angiográficamente significativas. El estudio Danish On-pump versus Off-pump Randomization Study (DOORS) ${ }^{18}$ y el meta-análisis de Takagi y cols ${ }^{19}$ son bien claros a este respecto. Takagi y cols ${ }^{19}$ aportan datos de diferentes cohortes que totalizan más de 100.000 pacientes. Por su parte, Sabik también ha incidido en el hecho muy bien conocido de que la eficacia de la CRM está directamente relacionada con la permeabilidad de los injertos construidos y con el número de los mismos, es decir, si la revascularización es completa o no ${ }^{20}$. Este es un idioma que debería ser entendido por todos. 


\section{La Definición}

El Diccionario de la Real Academia Española (RAE) en su Edición del Tricentenario contempla diversas acepciones de la palabra híbrido. Según la RAE y la Asociación de Academias de la Lengua Española (ASALE), un híbrido es un producto de elementos de distinta naturale$\mathrm{za}^{21}$. Por tanto, la revascularización miocárdica híbrida $(\mathrm{RMH})$ es una estrategia que incluye elementos diversos. La RMH conceptualmente persigue la combinación de las ventajas individuales de las dos estrategias principales, es decir, la CRM y la ICP. En cuanto a la CRM, la estrategia confirma un beneficio de supervivencia, basado, como ya se comentó, en la utilización del injerto de AMI izquierda en la DA y la asociación de doble AMI y revascularización arterial múltiple ${ }^{13-14-16}$. En lo que se refiere a la ICP, las ventajas se confinan en una menor agresión que la CRM con o sin CEC y una esperable y esperada superior tasa de revascularización mediante la utilización, más o menos discriminada, de dispositivos endocoronarios (conocidos como stents) de todo tipo, sin recubrimiento, liberadores de drogas o con bioandamio reabsorbible ${ }^{22}$. En definitiva, la RMH es una combinación del equipamiento del laboratorio de cateterismo cardiaco y de un quirófano reglado para intentar tratar cualquier lesión cardiovascular y en lo que respecta a este documento, la cardiopatía isquémica.

\section{Los estudios}

El primer estudio prospectivo y aleatorizado sobre RMH fue publicado por Gasior et al, en 2014 23 . Los autores hacían hincapié en las limitaciones de la CRM convencional y los resultados no satisfactorios a largo plazo de los injertos venosos para justificar la aleatorización a CRM o RMH. Los autores incluyeron 200 pacientes con enfermedad multivaso con afectación de la arteria coronaria descendente anterior (DA) y al menos una lesión llamada critica $(>70 \%)$ en otro vaso epicárdico mayor. Un aspecto llamativo fue la intención de conseguir revascularización completa de todos los vasos con diámetro superior a $2.0 \mathrm{~mm}$ y estenosis superiores por angiografía al $50 \%$. En el grupo RMH el tiempo medio entre el implante de AMI izquierda en la DA y la ICPO fue de 21 horas. El seguimiento se realizaba por angiografía a los 12 meses. El estudio confirmó que aproximadamente el $54 \%$ de los pacientes tenían enfermedad de tres vasos y la puntuación SYNTAX fue 23.4 en el grupo RMH y 22.8 en la de CRM. Se consiguió la revascularización planeada en el $93.9 \%$ de casos de RMH y $100 \%$ en la CRM. Se colocaron 2.3 dispositivos endocoronarios por paciente en el grupo RMH y el $85 \%$ de los pacientes del grupo de CRM fueron intervenidos sin CEC. La revascularización completa arterial se obtuvo en el $24 \%$ de los pacientes con una media de $2.7 \%$ de conductos implantados. En el grupo RMH se utilizó la técnica quirúrgica conocida en inglés como MIDCAB (Minimally invasive direct coronary artery bypass), lo cual no quedaba definido con claridad en la sección "Métodos", salvo la abreviatura en la Figura 1 del original. Hay que imaginar que MIDCAB era el acceso a la AMI izquierda a través de una toracotomía anterior izquierda de tamaño reducido, intervención restringida a subgrupos específicos de pacientes con seguimiento limitado. Uno de los estudios con mayor población de pacientes intervenidos con MIDCAB tiene asimismo, defectos metodológicos que los propios autores confirman ${ }^{24}$. En resumen, el estudio se dirigía a una población muy seleccionada, con puntuación baja de complejidad anatómica y prácticamente la mitad sin enfermedad de tres vasos. La tasa de conversión a estereotomía fue $6.1 \%$ en el grupo RMH. Un problema asociado desde el punto de vista metodológico es que la técnica quirúrgica se dejó a la preferencia del paciente, lo cual es un sesgo claro a pesar de que no hubo conversiones de CRM sin CEC a CRM con CEC. No hubo casos de accidente cerebrovascular en ninguno de los grupos.

Hay que resaltar que el seguimiento angiográfico a 12 meses no superó el $85 \%$ y que la permeabilidad del injerto de AMI izquierda fue del $93 \%$ en la CRM y del $94 \%$ en el grupo RMH con 5 casos de estenosis del injerto arterial en la DA por encima de $70 \%$, quizás en relación con que el $85 \%$ de los pacientes recibieron CRM sin CEC. La tasa de oclusión del dispositivo endocoronario fue $5.1 \%$ y la de estenosis mayor de $50 \%$ del dispositivo $7.5 \%$. Por último, recordar que no se incluyeron pacientes con lesión de tronco coronario izquierdo.

El estudio, a pesar del diseño con asignación aleatoria de grupos de tratamiento, tiene claras limitaciones. En primer lugar, los vasos diana debían tener más de $2.0 \mathrm{~mm}$. En la práctica quirúrgica, los vasos injertados suelen tener menos de $2.0 \mathrm{~mm}$ y eso es un factor importante a considerar desde el punto de vista de complejidad técnica y permeabilidad alejada de injertos o dispositivos. Los autores no justifican el tamaño de la muestra ya que es un estudio solo dirigido a evaluar la factibilidad del abordaje híbrido. En tercer lugar, la mayoría de pacientes, 85\% fue intervenido con el patrón de CRM sin CEC, lo cual añade un factor de confusión cuando la evidencia disponible lo primero que confirma en todos los estudios, es que la CRM sin CEC lleva asociada un menor número 
de injertos coronarios y eso ya quedo claro en el estudio DOORS ${ }^{18}$ y el meta-análisis de Takagi et al ${ }^{19}$ que ya se mencionan con anterioridad. El estudio tampoco está diseñado para detectar diferencias en mortalidad y el seguimiento es mínimo, solo de 12 meses, lo cual sabemos que no tiene valor a la hora de diferenciar eventos y permeabilidades. Por tanto, y solo con este estudio, por mucho que sea el primero prospectivo y aleatorizado sobre el tema, no se puede empezar a dictaminar que estamos delante de un cambio de paradigma en la CRM en los pacientes multivaso, tal y como de una forma muy probablemente precoz, aventurada e inapropiada, Puskas et al han dictaminado de forma radical ${ }^{25}$. Cuando solo se ha evaluado la factibilidad de un abordaje, por muy consensuado que sea, cuando hay dudas acerca del poder estadístico y sin seguimiento apropiado, es decir, por lo menos a cinco años, hay que evitar la radicalidad para no inducir confusión o practicas inadecuadas a la espera del acumulo de evidencia científica.

Las series retrospectivas de Shen $^{26}$ y Halkos ${ }^{27}$ sobre paciente intervenidos de forma hibrida por enfermedad multivaso y sobre tronco izquierdo ${ }^{28}$ adolecen de los mismos problemas, es decir, tamaños de muestra reducidos y recogidos en periodos de tiempo prolongados, de cinco años, los periodos de seguimiento son cortos, las tasas de re-revascularización son al menos cuatro veces superiores a la CRM sin CEC y, además, estos trabajos se basan fundamentalmente en estrategias de revascularización de CRM sin CEC que, como hemos dicho, todavía no es el patrón-oro en la CRM, tal y como apuntan también las guías de práctica clínica ${ }^{1}$.

Más recientemente, Modrau et $\mathrm{al}^{29}$ comunicaron el seguimiento angiográfico a un año de la RMH después del implante de un injerto de AMI izquierda en la DA a través de una hemiesternotomía inferior en un grupo de 100 pacientes consecutivos. El objetivo primario de eventos cardíacos y cerebrovasculares lo alcanzó el 20\% de los pacientes, 16 fueron re-revascularizados y hubo 5 re-intervenciones quirúrgicas durante la hospitalización inicial. Tras el alta, se realizó ICP en el 8\%. A 12 meses, la permeabilidad de la AMI izquierda fue del $98 \%$. El mensaje subyacente fue que la asociación de una permeabilidad de AMI izquierda elevada con un riesgo bajo de muerte y accidente cerebrovascular podría hacer que esta estrategia fuese prometedora en un futuro.

De esta apreciable serie se derivan dos problemas importantes, uno es la tasa de $20 \%$ de eventos adversos cardíacos y cerebrovasculares, inesperadamente alta y sin relación alguna con lo comunicado en el estudio SYNTAX a
1 y 5 años ${ }^{30-31}$. La revascularización repetida podría ser un argumento en este sentido cuya tasa fue del $16 \%$ en el año de seguimiento. El segundo problema, la re-revascularización inmediata fue muy superior a la comunicada por los propios autores en relación a la CRM con CEC, inferior al $1 \%^{29}$. Como en el estudio de Gasior et al ${ }^{23}$, este estudio tiene limitaciones importantes, en este caso la ausencia de un grupo control, la inclusión de pacientes de mínimo riesgo, la ausencia de lesiones coronarias complejas y el ser uni-institucional. En cualquier caso, la población del estudio de Modrau et $\mathrm{al}^{29}$ fue altamente seleccionada y el razonamiento del por qué los autores decidieron llevar a cabo este estudio considerando sus propios óptimos resultados con la CRM convencional es motivo de intriga.

Los recientes meta-análisis de Zhu et $\mathrm{al}^{32}$ y de $\mathrm{Hu}$ et $\mathrm{al}^{33}$ sobre la RMH y la CRM en la enfermedad multivaso y la de tronco izquierdo, como es lógico, no han podido aportar datos de peso aun recolectando la mayoría de información disponible en la actualidad. Los problemas de estos meta-análisis han sido, entre otros, la falta de estudios aleatorizados, ya que no pudieron incluir el estudio de Gasior et $\mathrm{al}^{23}$, la existencia de diversos factores de confusión, la existencia de sesgos de selección, tamaños de muestra muy reducidos, el diseño retrospectivo de todos los estudios con seguimientos muy cortos, sin estudio angiográfico de rutina y la mezcla de dispositivos endocoronarios liberadores de fármacos y de bioandamio reabsorbible.

Finalmente, el estudio reciente de Rosemblum et $\mathrm{al}^{34}$ que presenta el seguimiento de 300 pacientes intervenidos de RMH en un periodo de 10 años en comparación con una cohorte coetánea superior de más de 8000 pacientes, incluyendo un sofisticado estudio estadístico con análisis de propensión, confirma que la RMH podría representar una alternativa segura y menos invasora que la CRM convencional en pacientes muy cuidadosamente seleccionados ya que sus resultados a corto plazo fueron similares a la CRM con injerto simple o doble de AMI. El entusiasmo desatado a raíz de este estudio queda, no obstante, matizado y temperado por la necesidad de proponer estudios prospectivos y aleatorizados con diseño y tamaños de muestra adecuados ${ }^{35}$, lo cual no parece sencillo en el momento actual, considerando las necesidades financieras y logísticas intrínsecas y la eventual dificultad de encontrar un patrocinador.

Por tanto, en el momento actual la base científica para el soporte incondicional de la RMH es aun, limitada a la espera de seguir recopilando información proveniente de 
estudios de calidad que puedan contrastarse con la información acumulada en los últimos cincuenta años.

\section{Los comentarios adicionales}

Antes de concluir y visto lo disponible en la literatura acerca de la RMH, hay que plantearse diversas consideraciones que inciden en la técnica, la estrategia y su aplicabilidad de forma generalizada, a saber:

\section{Selección del paciente}

Los pacientes con limitaciones para CRM como calcificación, vasos de pobre calidad, la falta de conductos, una cavidad torácica grande, la ventilación un pulmonar durante el procedimiento, hipertensión pulmonar y comorbilidades y eventuales contraindicaciones han de ser tenidas en cuenta. Estos aspectos no suelen reflejarse en la literatura por la muy elevada selección de los pacientes en las cortas series comunicadas.

\section{La secuencia de actuación}

Hay que plantearse si el momento de la parte quirúrgica o la parte intervencionista tienen o tendrán influencia en los resultados. No queda claro en la literatura, de forma fehaciente, por la escasez de datos, cuál es la estrategia adecuada. Parece que la decisión sigue siendo individual en 2016 y puede contemplar tres escenarios: a) Estadio único con intervención quirúrgica e ICP simultánea en el mismo acto, b) Estadio doble con ICP previa a la CRM, y c) Estadio doble con CRM previa a la ICP. Y por último, no queda claro cuándo deben realizarse. En el estudio de Gaser et $\mathrm{al}^{23}$ el único prospectivo y aleatorizado, e intervalo mediano entre el implante de AMI izquierda y la ICP fue de 21 horas. ¿Es este el intervalo idóneo? ¿Si no lo es, cuál es? Hay variabilidad en la escasa literatura y, por tanto, este factor tiempo puede tener influencia, pero en el momento presente no sabemos si la tiene y cuál.

\section{La técnica quirúrgica}

La RMH es, por definición, un abordaje de mínima invasión o lo pretende. Y de nuevo, considerando las tendencias actuales, algunas ya en el mercado quirúrgico desde hace unas dos décadas, ¿cuál es la que debe confirmarse como de elección? En el estudio de Gaser et al $^{23}$, el implante de AMI se realizó con acceso MIDCAB, es decir, incisión limitada antero lateral izquierda. La cirugía conocida en terminología inglesa como EndoACAB (Endoscopic atraumatic coronary artery bypass), que empezó a conocerse hace más de 10 años $^{36}$, no parece haber despegado de forma definitiva. Y de forma inesperada, parece que podría tener una respuesta funcional de ejercicio inferior a la prevista según datos recientes ${ }^{37}$. EndoA$\mathrm{CAB}$ requiere una experiencia técnica no despreciable y su versión actualizada, TECAB (Totally endoscopic coronary artery bypass) utilizando asistencia robótica, es una opción de elevado coste y alta complejidad, todavía al alcance de unos pocos, con escasos datos de calado en la literatura ${ }^{38-39}$ y con seguimiento corto, por debajo de 5 años. No hay que olvidar, en ningún caso, la prolongada curva de aprendizaje que estas opciones quirúrgicas llevan asociadas y sobre la que no hay información en la literatura.

\section{La terapia antitrombótica}

Al representar la RMH la asociación de un procedimiento quirúrgico y una ICP, es obligado considerar la terapia antitrombótica por el implante de dispositivos endocoronarios. Para empezar, todavía no hay recomendaciones o guías de práctica disponibles, por la escasa información disponible tal y como ya se comentó. Por ahora no hay estudios prospectivos planteados sobre la base de esta necesaria terapia y no hay acuerdo generalizado acerca de doble terapia basada en ácido acetil salicílico y clopidogrel o si deberían incluirse inhibidores de la Glicoproteina IIb/IIIa o nuevos anticoagulantes.

\section{Los comentarios finales}

Entendiendo que, como híbrido ${ }^{21}$, la RMH incorporaría lo mejor de cada mundo, el quirúrgico y el de la ICP, la información que se maneja en la actualidad proviene de décadas de experiencia acumulada por multitud de autores que han conseguido que la CRM cumpla un objetivo primordial, que es la reproducibilidad con resultados inmediatos, a corto y a largo plazo, por encima de quince años de seguimiento, satisfactorios para los pacientes, los médicos tratantes y los cirujanos responsables ${ }^{4-5}$. El patrón-oro es, por el momento, la CRM tal y como queda reflejado en las guías conjuntas médico-quirúrgicas de práctica clínica ${ }^{1}$. Drinkwater ya comentó en un elegante análisis ${ }^{40}$ de los datos de seguimiento de SYNTAX a 5 años ${ }^{41}$ con supervivencias similares en los grupos de injerto arterial único o múltiple, a la espera de poder incorporar más información en el futuro si se completa un seguimiento más alejado aun teniendo en cuenta las contribuciones que reafirman el papel del injerto arterial múltiple ${ }^{13-17}$. Y finalmente, las juiciosas palabras de Sabik $^{42}$ resaltan de nuevo el hecho bien conocido de que la eficacia y eficiencia de la CRM convencional con CEC 
está directamente relacionada con la permeabilidad de los injertos y lo completa que sea la revascularización. En un comentario previo ${ }^{43}$ subrayábamos que la $\mathrm{RMH}$, a pesar de la información recogida en la última década, si bien escasa, podría ser considerada en un futuro como una alternativa prometedora a la CRM y la ICP por las ventajas que se esperan de ella, pero confirmando un camino aun largo a recorrer. Sin embargo, hay variables que hay que analizar con detenimiento como los costes, la logística, la complejidad y la curva de aprendizaje, además de la obligatoriedad de realizar estudios bien diseñados con aleatorización de grupos de tratamiento y calidad en el seguimiento que no debería bajar de 10 años para poder compararse con la estrategia convencional. Pasado un tiempo y con la incorporación de información adicional, en estos momentos se puede decir que la RMH nos aporta poco todavía y que tiene por delante ese largo camino a recorrer si pretende compararse con el patrón-oro que conocemos en la actualidad.

\section{Referencias}

1. KOLH P, WINDECKER S, ALFONSO F, COLLET JP, CREMER J, FALK V, et al; Task Force on Myocardial Revascularization of the European Society of Cardiology and the European Association for Cardio-Thoracic Surgery; European Association of Percutaneous Cardiovascular Interventions. 2014 ESC/EACTS Guidelines on myocardial revascularization: the Task Force on Myocardial Revascularization of the European Society of Cardiology (ESC) and the European Association for Cardio-Thoracic Surgery (EACTS). Developed with the special contribution of the European Association of Percutaneous Cardiovascular Interventions (EAPCI). Eur J Cardiothorac Surg 2014; 46:517-592.

2. MOHR FW, MORICE MC, KAPPETEIN AP, FELDMAN TE, STÅHLE E, COLOMBO A, et al. Coronary artery bypass graft surgery versus percutaneous coronary intervention in patients with three-vessel disease and left main coronary disease: 5-year follow-up of the randomised, clinical SYNTAX trial. Lancet 2013; 381:629-638.

3. MACK M, BAUMGARTEN H, LYTLE B. Why surgery won the SYNTAX trial and why it matters. J Thorac Cardiovasc Surg 2016 May 5.
4. LOOP FD, LYTLE BW, COSGROVE DM, STEWART RW, GOORMASTIC M, WILLIAMS GW, et al. Influence of the internal-mammary-artery graft on 10-year survival and other cardiac events. N Engl J Med 1986;314:1-6.

5. LOOP FD. Coronary artery surgery: the end of the beginning. Eur J Cardiothorac Surg 1998;14:554-571.

6. KOWALEWSKI M, PAWLISZAK W, MALVINDI PG, BOKSZANSKI MP, PERLINSKI D, RAFFA GM et al. Off-pump coronary artery bypass grafting improves short-term outcomes in high-risk patients compared with on-pump coronary artery bypass grafting: Meta-analysis. J Thorac Cardiovasc Surg 2015; 151:60-77.

7. TAGGART DP. Contemporary coronary artery bypass grafting. Front Med 2014; 8:395-398.

8. SPENCER FC, YONG NK, Prachuabmoh K. Internal mammary-coronary artery anastomoses performed during cardiopulmonary bypass. J Cardiovasc Surg (Torino) 1964; 5:292-297.

9. FAVALORO RG, EFFLER DB, GROVES LK, SONES FM 
JR, FERGUSSON DJ. Myocardial revascularization by internal mammary artery implant procedures. Clinical experience. J Thorac Cardiovasc Surg 1967; 54:359-370.

10. WEINTRAUB WS, GRAU-SEPULVEDA MV, WEISS JM, O'BRIEN SM, PETERSON ED, KOLM P, et al. Comparative effectiveness of revascularization strategies. N Engl J Med 2012; 366:1467-1476.

11. FARKOUH ME, DOMANSKI M, SLEEPER LA, SIAMI FS, DANGAS G, MACK M, et al. Strategies for multivessel revascularization in patients with diabetes. N Engl J Med 2012; 367:2375-2384.

12. EPSTEIN AJ, POLSKY D, YANG F, YANG L, Groeneveld PW. Coronary revascularization trends in the United States, 20012008. JAMA 2011; 305:1769-1776.

13. LYTLE BW, BLACKSTONE EH, LOOP FD, HOUGHTALING PL, ARNOLD JH, AKHRASS R, et al. Two internal thoracic artery grafts are better than one. J Thorac Cardiovasc Surg 1999; 117:855-872

14. LOCKER C, SCHAFF HV, DEARANI JA, JOYCE LD, PARK SJ, BURKHART HM, et al. Multiple arterial grafts improve late survival of patients undergoing coronary artery bypass graft surgery: analysis of 8622 patients with multivessel disease. Circulation 2012; 126:1023-1030.

15. BUXTON BF, SHI WY, TATOULIS J, FULLER JA, ROSALION A, HAYWARD PA. Total arterial revascularization with internal thoracic and radial artery grafts in triple-vessel coronary artery disease is associated with improved survival. J Thorac Cardiovasc Surg 2014; 148:1238-1243.

16. LOCKER C, SCHAFF HV, DALY RC, DEARANI JA, BELL MR, FRYE RL, et al. Multiple arterial grafts improve survival with coronary artery bypass graft surgery versus conventional coronary artery bypass grafting compared with percutaneous coronary interventions. J Thorac Cardiovasc Surg 2016; 152:369-379.

17. KURLANSKY P. Multiple arterial grafting: Please don't confuse me with the facts. J Thorac Cardiovasc Surg 2016; 152:380-381 .

18. HOULIND K, FENGER-GRØN M, HOLME SJ, KJELDSEN BJ, MADSEN SN, RASMUSSEN BS, et al; DOORS Study Group. Graft patency after off-pump coronary artery bypass surgery is inferior even with identical heparinization protocols: results from the Danish On-pump Versus Off-pump Randomization Study (DOORS). J Thorac Cardiovasc Surg 2014; 148:1812-1819.

19. TAKAGI H, UMEMOTO T; ALL-Literature Investigation of Cardiovascular Evidence (ALICE) Group. Worse long-term survival after off-pump than on- pump coronary artery bypass graf- ting. J Thorac Cardiovasc Surg 2014;148: 1820-1829.

20. SABIK JF III. On-pump coronary revascularization should be our preferred surgical revascularization strategy. J Thorac Cardiovasc Surg 2014; 148:2472-2474.

\section{WWW.RAE.ES}

22. MURAMATSU T, ONUMA Y, ZHANG YJ, BOURANTAS CV, KHARLAMOV A, DILETTI R et al. Progress in treatment by percutaneous coronary intervention: the stent of the future. Rev Esp Cardiol (Engl Ed). 2013; 66:483-496.

23. GĄSIOR M, ZEMBALA MO, TAJSTRA M, FILIPIAK K, GIERLOTKA M, et al; POL-MIDES (HYBRID) Study Investigators. Hybrid revascularization for multivessel coronary artery disease. JACC Cardiovasc Interv 2014; 7:1277-1283.

24. HOFFMANN G, FRIEDRICH C, BARRABAS M, PETZINA R, HANEYA A, PANHOLZER B, et al. Short- and long-term follow-up after minimally invasive direct coronary artery bypass in octogenarians. Interact Cardiovasc Thorac Surg. 2016;23:377-82.

25. PUSKAS JD, PAWALE A, SHARMA SK. Hybrid coronary revascularization: a new treatment paradigm for selected patients with multivessel coronary artery disease. JACC Cardiovasc Interv 2014; 7:1284-1286.

26. SHEN L, HU S, WANG H, XIONG H, ZHENG Z, LI L, et al. One-stop hybrid coronary revascularization versus coronary artery bypass grafting and percutaneous coronary intervention for the treatment of multivessel coronary artery disease: 3-year follow-up results from a single institution. J Am Coll Cardiol 2013; 61:2525-2533.

27. HALKOS ME, VASSILIADES TA, DOUGLAS JS, MORRIS DC, RAB ST, LIBERMAN HA, et al. Hybrid coronary revascularization versus off-pump coronary artery bypass grafting for the treatment of multivessel coronary artery disease. Ann Thorac Surg 2011; 92:1695-1701.

28. HALKOS ME, RAB ST, VASSILIADES TA, MORRIS DC, DOUGLAS JS, KILGO PD, et al. Hybrid coronary revascularization versus off-pump coronary artery bypass for the treatment of left main coronary stenosis. Ann Thorac Surg 2011; 92:2155-2160.

29. MODRAU IS, HOLM NR, MÆNG M, BØTKER HE, CHRISTIANSEN EH, KRISTENSEN S, et al. Hybrid Coronary Revascularization Study Group. One-year clinical and angiographic results of hybrid coronary revascularization. J Thorac Cardiovasc Surg 2015; 150:1181-1186.

30. SERRUYS PW, MORICE MC, KAPPETEIN AP, COLOMBO 
A, HOLMES DR, MACK MJ, et al. Percutaneous coronary intervention versus coronary-artery bypass grafting for severe coronary artery disease. N Engl J Med. 2009; 360:961-72.

31. HEAD SJ, DAVIERWALA PM, SERRUYS PW, REDWOOD SR, COLOMBO A, MACK MJ, et al. Coronary artery bypass grafting vs. percutaneous coronary intervention for patients with three-vessel disease: final five-year follow-up of the SYNTAX trial. Eur Heart J. 2014; 35: 2821-30.

32. ZHU P, ZHOU P, SUN Y, GUO Y, MAI M, ZHENG S. HYBRID coronary revascularization versus coronary artery bypass grafting for multivessel coronary artery disease: systematic review and meta-analysis. J Cardiothorac Surg 2015;10:63.

33. HU FB, CUI LQ. Short-term clinical outcomes after hybrid coronary revascularization versus off-pump coronary artery bypass for the treatment of multivessel or left main coronary artery disease: a meta-analysis. Coron Artery Dis 2015; 26:526-534.

34. ROSENBLUM JM, HARSKAMP RE, HOEDEMAKER N, WALKER P, LIBERMAN HA, DE WINTER RJ, et al. Hybrid coronary revascularization versus coronary artery bypass surgery with bilateral or single internal mammary artery grafts. J Thorac Cardiovasc Surg 2016; 151:1081-1089.

35. HIESINGER W, ATLURI P. Hybrid coronary revascularization: Ready for prime time, but who should star? J Thorac Cardiovasc Surg 2016; 151:1090-1091.

36. VASSILIADES TA JR, REDDY VS, PUSKAS JD, GUYTON RA. LONG-TERM results of the endoscopic atraumatic coronary artery bypass. Ann Thorac Surg 2007; 83:979-984.
37. HANSEN D, ROIJAKKERS R, JACKMAERT L, ROBIC B, HENDRIKX M, YILMAZ A, et al. Compromised Cardiopulmonary Exercise Capacity in Patients Early After Endoscopic Atraumatic Coronary Artery Bypass Graft: Implications for Rehabilitation. Am J Phys Med Rehabil. 2016 May 12. [Epub ahead of print]

38. BONAROS N, SCHACHNER T, LEHR E, KOFLER M, WIEDEMANN D, HONG P, et al. Five hundred cases of robotic totally endoscopic coronary artery bypass grafting: predictors of success and safety. Ann Thorac Surg 2013; 95:803-812.

39. BONAROS N, SCHACHNER T, KOFLER M, LEHR E, LEE J, VESELY M, et al. Advanced hybrid closed chest revascularization: an innovative strategy for the treatment of multivessel coronary artery disease. Eur J Cardiothorac Surg 2014; 46:e94102 .

40. DRINKWATER DC JR. Multiple arterial grafting: stay the course. J Thorac Cardiovasc Surg. 2015; 150: 452-453.

41. PARASCA CA, HEAD SJ, MOHR FW, MACK MJ, MORICE MC, HOLMES DR JR, et al; SYNTAX Investigators. The impact of a second arterial graft on 5-year outcomes after coronary artery bypass grafting in the Synergy Between Percutaneous Cor- onary Intervention With TAXUS and Cardiac Surgery Trial and Registry. J Thorac Cardiovasc Surg. 2015; 150: 597-606.e2.

42. SABIK JF III. On-pump coronary revascularization should be our preferred surgical revascularization strategy. J Thorac Cardiovasc Surg 2014; 148: 2472-2474. 